\title{
Interoperabilidad en la gestión documentaria en el sector público
}

María Elizabeth Pérez Vásquez eliperezvasquez@gmail.com Universidad Cesar Vallejo- Perú

Julio Manuel Rodríguez Vásquez jrodriguez.contadores@ gmail.com Universidad Cesar Vallejo- Perú

Lin Álvarez Ríos Linalvarez10@hotmail.com Universidad Cesar Vallejo- Perú

Gabriela del Pilar Palomino Alvarado dpalominoal@ucvvirtual.edu.per Universidad Cesar Vallejo- Perú

\section{RESUMEN}

Los gobiernos perciben la importancia del uso y desarrollo de la tecnología para mejorar su gestión interna y facilitar la atención al ciudadano, basado en el entorno digital para el acceso a los servicios, soportado internamente en la interoperabilidad como un término referido a la capacidad para compartir información entre organismos con infraestructura tecnológica y herramientas similares. Por esta razón se han desarrollado arquitecturas digitales para establecer guías que se ajusten a modelos como los portales de interoperabilidad en la gestión documentaria. El objetivo del estudio, es realizar una revisión sistemática de artículos científicos sobre el tema, disponible en la base de datos EBSCO, Scopus, SciELO, ProQuest y Redalyc en español e inglés de los últimos 5 años. Método: se ingresó a las bases de datos realizándose la búsqueda del término "interoperabilidad", "gestión documentaria", descartándose artículos que no son arbitradas y académicas y publicados antes del año 2017; como resultado de ello se evidenció que existe la necesidad urgente de implementar la Plataforma de interoperabilidad del Estado para los sistemas de trámite documentario, servicios públicos en línea por medios electrónicos, el intercambio electrónico de datos entre entidades del Estado a través de internet; concluyendo que el gobierno electrónico y la interoperabilidad aún siguen siendo incipientes en nuestro país, donde las entidades deben aplicar la política en gestión documentaria y el compromiso de la alta dirección para su implementación.

Palabras clave: Interoperabilidad., gestión documental., plataforma., políticas. 


\title{
Interoperability in documentary management in the públic sector
}

\begin{abstract}
Governments perceive the importance of the use and development of technology to improve their internal management and facilitate citizen service, based on the existence of a digital environment for access to services, internally supported by interoperability as a term referring to the ability to share information between organizations with technological infrastructure and similar tools. For this reason, digital architectures have been developed to establish guides that conform to models such as interoperability portals in document management. The objective of the study is to carry out a systematic review of scientific articles on the subject, available in the EBSCO, Scopus, SciELO, ProQuest and Redalyc databases in Spanish and English for the last 5 years. Method, databases were entered, searching for the term "interoperability", "document management", discarding articles that were not refereed and academic and published before 2017; As a result, it was evidenced that there is an urgent need to implement the State's interoperability platform for documentary processing systems, online public services by electronic means, and the electronic exchange of data between State entities through the Internet; concluding that electronic government and interoperability are still incipient in our country, where entities must apply the policy on document management and the commitment of senior management for its implementation.
\end{abstract}

Keywords: Interoperability., document., management., platform., policies.

Artículo recibido: 05 de Abril 2021 Aceptado para publicación: 28 de Mayo 2021 Correspondencia: eliperezvasquez@gmail.com Conflictos de Interés: Ninguna que declarar 


\section{INTRODUCCIÓN}

En los últimos años el avance tecnológico, electrónico y digital ha avanzado en el mundo, impulsando una administración pública moderna con transparencia en la gestión a través de la simplificación administrativa, para agilizar y reducir el tiempo de dichos procesos y con el uso de documentos electrónicos, con el objetivo de favorecer a los ciudadanos el acceso a los servicios públicos, y obtener una administración eficaz y eficiente; aplicando una política de uso de documentos electrónicos y de la aplicabilidad de normas internacionales y buenas prácticas (Perpinya, 2018).

El gobierno digital consiste en ofrecer nuevas formas de valor público y hacer que los servicios y los procedimientos gubernamentales sean de diseño digital por esencia, lo que exige la integración de las Tecnologías de la Información y la Comunicación (TIC) en la agenda de la reforma del sector público desde su concepción (OCDE, 2016). La evolución del gobierno digital, se ve directamente afectada por el grado de desarrollo de la interoperabilidad, considerada como un pilar fundamental para desarrollar servicios eficientes, eficaces, simplificados y confiables para el ciudadano bajo una visión unificada; a fin de desarrollar capacidades para el intercambio fácil, seguro y transparente de la información entre los organismos del poder público, poder popular y/o entidades privadas que tributen a la prestación de los servicios al colectivo (Araujo, et. al, 2020).

De acuerdo al Decreto Supremo 083-2011-PCM, la interoperabilidad, es comprendida como el intercambio de datos, información y conocimiento entre instituciones públicas de los diferentes niveles de gobierno, que posibilita la cooperación entre las instituciones públicas, sin distinción del nivel de desarrollo tecnológico; facilita la simplificación de procesos administrativos, de comunicación, y de negociación, también permite al Estado ofrecer al ciudadano servicios y trámites ágiles y de bajo costo, además elimina el uso de documentación física y garantiza la seguridad de los datos de los usuarios. Además, se crea la Plataforma de Interoperabilidad del Estado (PIDE) que es una infraestructura tecnológica que permite la implementación de servicios públicos por medios electrónicos y el intercambio electrónico de datos, entre entidades del estado a través de internet, telefonía móvil y otros medios tecnológicos disponibles.

Asimismo, la Presidencia de Concejos de Ministros del Perú a través de la política nacional de modernización de la gestión pública al 2021, la plataforma de 
interoperabilidad debe permitir interactuar y gestionar la diversidad de componentes con la utilización de diversos productos de múltiples proveedores en diferentes organizaciones, de forma que cada institución pueda obtener la información previamente definida y estandarizada que le sirva de base para implementar en forma masiva servicios públicos en línea a través de portales en Internet y telefonía móvil, en el cual se debe diseñar e implementar bases y almacenes de datos integrados a nivel nacional y a través de todas las entidades públicas para también facilitar el intercambio de información para tomar decisiones en torno a la gestión institucional, aspectos de planificación, seguimiento y evaluación; por este motivo el Estado busca herramientas de las TICs, la digitalización de los sistemas, procesos y documentos.

$\mathrm{Al}$ respecto, el Poder Ejecutivo emitió el Decreto Legislativo 1310 con el que se aprueban medidas adicionales para la simplificación de procesos administrativos; en el artículo 8 de dicho decreto, el Estado aprueba la implementación de la PIDE para la interoperabilidad de los sistemas de trámite documentario; pese a que se propuso su implementación en el DS 83-2011/PCM en el 2011 éste no se ha ejecutado; ahora, el objetivo normativo es que funcione la "interoperabilidad" entre todas las entidades del Estado, dando pie a la modernización de sus sistemas documentales, es decir, la gestión documental.

Por otro lado, a través de la Resolución de Secretaría del Gobierno Digital N 001-2017PCM/SEGD, aprueba el Modelo de Gestión Documental enmarcado en el Decreto Legislativo 1310, señalando que su implementación iba de la mano con la fecha de implementación de la PIDE en las entidades gubernamentales, es decir, el 31 de diciembre del 2018; sin embargo, la gestión documentaria en el sector público es deficiente en nuestro país, pese a las múltiples normas aprobadas y mucho más en la actualidad durante la pandemia del Covid-19, ha permitido que se aceleren algunos aspectos como la implementación de la mesa de partes virtual y el uso de la firma digital; en el cual la interoperabilidad no funciona entre las entidades del Estado, existiendo la necesidad de que las instituciones cuenten con una política en gestión documental para el intercambio de información digital y utilizar la información que las entidades generan para retroalimentar otras bases de datos; y el compromiso de la alta dirección para su implementación para lograr la transformación digital. (Alarcón, 2020) 
El objetivo, de esta investigación es realizar un análisis sobre la aplicabilidad del portal PIDE y la difusión de la gestión documental para la transparencia, la interoperabilidad y la orientación a los usuarios.

Donde para esto el gobierno español establece con la Ley 11/2007 del 22 de junio, el ámbito de aplicabilidad y como un derecho de los ciudadanos a interrelacionar con medios electrónicos con sus entidades públicas, publicado en el diario oficial del gobierno de España. (Agencia Estatal Boletín Oficial del Estado). En Latinoamérica y el Caribe la mayoría de países han establecidos lineamientos referidos al gobierno electrónico y así promover la eficacia, eficiencia y transparencia en las administraciones públicas, uno de los impulsores o iniciadores de la simplificación administrativa con el uso de documentos electrónicos en el gobierno de Colombia, que cuenta con un Ministerio de Tecnologías de Información, el impulso es alto, consecuencia de la transformación en el no uso del papel, tanto así que se podría considerar que son un Estado digitalizado, según lo normado con la Directiva Presidencial No 04 del 2012, que en su lineamiento 3, precisa que en el lapso de 12 meses los organismos de la administración pública, deberán reemplazar el uso de papel en sus comunicaciones internas, por soportes electrónicos. (Ministerio de Tecnologías de la Información y las Comunicaciones-MinTic, 2012), a diferencia de los demás países latinoamericanos, Colombia tiene a su ente rector en modernización del Estado en un nivel ministerial; las instituciones estatales orientan el uso de sistemas de gestión documental, que con una política "cero papel”, haga uso de un trámite con documentos digitalizados o electrónicos. (Castaño, 2017)

La importancia que tienen el gobierno digital y la tecnología para cumplir con los objetivos de gobernanza deseados en los próximos años, se visualiza en países en desarrollo, desafiando a países desarrollados en estas áreas. La tendencia cambiante ha comenzado, el aumento de naciones y políticos que defienden la causa del gobierno electrónico son indicadores positivos. Es importante destacar que, como marco de trabajo de las diferentes instituciones gubernamentales, el gobierno digital es esencial para lograr la cuarta revolución industrial y aquellas que no trabajen en estos temas se quedarán obsoletas (Toro, et. Al, 2019)

\section{ESTRATEGIAS METODOLÓGICAS O MATERIALES Y MÉTODOS}

La presente es una investigación de revisión, se utilizaron métodos como: el análisis síntesis, inducción-deducción, de lo general a lo particular, es decir se procedió con la 
búsqueda de referentes que permitieran establecer los principales aspectos a tener en cuenta, luego se hizo la revisión de diferentes investigaciones cuyo foco es el tema de este trabajo.

Dentro de los métodos se utilizó: la observación y el análisis documental, cuyos resultados fueron: se seleccionaron 30 artículos, de las cuales solo se eligieron a 28 artículos teniendo en cuenta las similitudes de la temática, cuyo país de procedencia fueron: Colombia, Cuba, Brasil, Ecuador, México, Habana, Perú, Argentina y España, inicialmente fue necesario determinar las pautas de búsqueda para la revisión empleando los términos gobierno electrónico, gobierno digital, interoperabilidad, gestión documentaria, seguridad y privacidad de la información, indexados, de esta forma, fue más fácil y pertinente encontrar la información usando los recursos Google Académico, EBSCO, Science Direct, Redalycs, Scielo, Scopus y ProQuest en el intervalo mencionado, de esta manera, se obtuvieron los aspectos listados para tener una idea de la tendencia en la implementación del gobierno electrónico con la interoperabilidad de la gestión documentaria.

\section{RESULTADOS Y DISCUSIÓN}

Según Febles y Gonzales (2020) señalan que la interoperabilidad y la estandarización de los datos constituyen temas prioritarios que hará que el gobierno electrónico actúe como tal y no se obstaculice la construcción de un sector público basado en datos, en ese contexto definen que la interoperabilidad es el ejercicio de colaboración entre organizaciones para intercambiar información y conocimiento en el marco de sus procesos de negocio, con el propósito de facilitar la entrega de servicios en línea a ciudadanos, empresas y a otras entidades, considerando cuatro (4) niveles: Organizacional, Semántico, Técnico y Legal, donde fueron evaluados por expertos en derecho informático teniendo en cuenta publicaciones sobre el tema, experiencias internacionales y las características propias de Cuba, como conceptualización de un esquema nacional de interoperabilidad Cubano (ENIC), no existe hasta la fecha un modelo integrador que dé cuenta del marco teórico conceptual de cómo evaluar el avance en gobierno electrónico y en específico de la interoperabilidad, tampoco se ha desarrollado una arquitectura que lo contemple; en el cual proponen la implementación del ENIC, que contemple tres componentes fundamentales: Tipología, Gobernanza y una Plataforma de interoperabilidad con el objetivo de soportar la estrategia de Cuba de 
proveer servicios electrónicos centrados en el usuario, facilitando los servicios y sistemas entre administraciones públicas, así como entre administraciones y el público a nivel nacional.

Para De Grande (2020) en su investigación sobre gobierno electrónico y accesibilidad: disponibilidad de servicio en plataformas online estatales de la argentina, definen en tres categorías: Ministerios nacionales, Organismos estatales y Universidades nacionales, en el cual la primera categoría reúne sitios institucionales de una selección de ministerios nacionales; los cuales estarían radicados en la Ciudad de Buenos Aires y estos servidores pueden o no compartir su infraestructura de datos, hecho que debería evidenciarse en los rendimientos similares o disímiles de sus contenidos; en la segunda categoría incluye sitios de organismos dependientes de la administración pública nacional, los cuales si bien dependen de ministerios nacionales, podrían tener un tipo de respuesta diferenciado debido a la mayor complejidad de los servicios que proveen.

Mientras que los sitios institucionales de ministerios nacionales muestran contenidos usualmente 'estáticos', los sitios fichados como organismos nacionales ofrecen sistemas de reserva de turnos para la gestión de trámites personales o la gestión de trámites online; tanto de tipo tributario, de acceso a la seguridad social, así como la solicitud de subsidios entre otros. La tercera categoría reúne a Universidades nacionales, las cuales a diferencia de los dos grupos de sitios anteriores agregan a su disponibilidad el desafío de la distribución geográfica, a pesar de la organización nacional de tales universidades, su funcionamiento autónomo hace esperable que sus servidores de contenidos repliquen la distribución geográfica de estas sedes académicas; de este modo, los tres grupos esperan representar realidades diferentes de la infraestructura estatal nacional, siendo los dos primeros grupos servidores dependientes de organismos centralizados, y el tercer grupo un conjunto de instituciones distribuidas en el territorio. En este sentido, como primer resultado, emerge el hecho de que en los sitios dependientes del Estado nacional la cantidad de pedidos fallidos cada mil solicitudes duplica lo observado en las categorías no dependientes del Estado nacional. Mientras que en las demás categorías los sitios no superan en promedio las 10 fallas por cada mil pedidos (con excepción de proveedoras de servicios públicos, las cuales dependen del control del Estado nacional), en las tres categorías de sitios dependientes del Estado nacional las fallas se encuentran por encima de los 23 pedidos por cada mil; es decir, que los sitios dependientes del Estado nacional 
mostraron un rendimiento muy inferior en comparación con los demás sitios incluidos como referencia, ya fueran estos medios de prensa, de comercio electrónico o páginas de la administración de la Ciudad de Buenos Aires.

De acuerdo (Sant, et. Al, 2020) determinan que el problema público a través de la plataforma de interoperabilidad del Estado (PIDE) tiene relación con la satisfacción ciudadana, elevación de los niveles de satisfacción y gobierno digital, en el cual abordan dos problemas el primero con el nivel de interés o la baja adherencia de las municipalidades distritales de Lima a la Plataforma de Interoperabilidad del Estado en el periodo de 2016-2018; y el segundo con el problema a nivel de producto o reducida cobertura de la Plataforma de Interoperabilidad del Estado desarrollada por la Oficina Nacional de Gobierno Electrónico e Informática (ONGEI) en las municipalidades distritales de Lima en los años 2016-2018.

Asimismo, la obtención de información fue adoptar como punto de partida un territorio que presentara las condiciones inicialmente necesarias para adherirse al PIDE; y que estén vinculados al mismo distrito porque sería una forma de entender cómo se establecieron los procesos de comunicación entre los órganos e identificar formas de replicar el modelo en otras localidades; sin embargo, no se tomaron en cuenta los problemas de implementación de bienes y servicios para mejorar los efectos de las políticas para el cumplimiento de la adhesión a la plataforma, también las organizaciones no tienen práctica común y la interoperabilidad requiere una fuerte capacidad político-relacional de las organizaciones para aprender a trabajar de manera integrada como es el caso, de la administración privada; la gran mayoría de las instituciones que ofrecen atención al público no cuentan con funcionarios capacitados en el uso de la PIDE, un porcentaje importante se resiste a emplear por la brecha digital o porque temen ser reemplazados por ella; asimismo, no hay interés de parte de los directivos en hacerlo, las políticas públicas es importante considerar la participación social y escuchar a los actores como un elemento fundamental para permitir políticas públicas eficaces y eficientes que generen un valor público que permita la satisfacción y la confianza de la sociedad en el gobierno.

La interoperabilidad y la estandarización de los datos constituyen temas prioritarios que hará que el Gobierno electrónico actúe como tal y no se obstaculice la construcción de un sector público basado en datos. Una solución a esto, son los esfuerzos para digitalizar el sector público y la coordinación que ello implica a nivel nacional, evitar la fragmentación 
digital de los trámites, los servicios y los datos. En este contexto se impone la Interoperabilidad como uno de los elementos claves para avanzar en las etapas de Gobierno electrónico establecidas en Cuba

Por otro lado, para Cusihuamán, et al., (2020) enfatiza que las tecnologías de información y comunicación, interculturalidad y desarrollo rural en la provincia de la unión, Arequipa y Perú, las consideraciones prácticas han mostrado experiencias de aplicación de tecnologías de la información y comunicación, sin tener en cuenta propuestas integrales, por lo que, terminan siendo experimentos fallidos.

A esto se suma que, en el modelo de gobierno electrónico en el Perú, los servicios para el ciudadano en los portales de los gobiernos regionales los resultados a las tres categorías son: categoría de calidad de interacción, oscilan entre 12,20 a 14,24 de calificación; categoría de calidad de la información oscilan entre 23,73 a 25,42 de calificación y categoría de medio digital oscilan entre 24,15 a 27,68, de calificación. (Alvarado, et al; 2019)

Sin embargo, en el gobierno de México los portales web, si cumplen con las seis etapas: presencia, información, interacción, transacción, integración y participación política, se identificó de los 51 municipios revisados, 27 no cuentan con página electrónica y 24 si tiene página del municipio, así mismo solo 20 portales estaban activos, 4 de ellos marcaban como inactivos o en mantenimiento. (Arango, 2018)

En La Habana, la Gestión Documental como buena práctica para reducir el consumo de papel realizaron el cálculo de la cantidad del uso de hojas para la realización de pruebas parciales y totales acumulándose como evidencias en promedio entre el Curso Regular Diurno y el Curso por Encuentro un total de 186900 hojas cada año, lo que significa que para producir esa cantidad de hojas se necesitarían 22 árboles lo que resultan 1,30 toneladas de papel y un árbol contribuye a la respiración de 3 personas en un día y una tonelada a 57 personas, considerando aspectos positivos y negativos de la gestión de documentos y su participación de manera directa e indirecta en la contaminación ambiental; así como el ahorro, en la introducción de la gestión documental en el desarrollo de los procesos institucionales.(López, 2019)

El Gobierno digital para la construcción de Estados más transparentes y proactivos en Colombia, Toro (2019) considera que las TIC se han convertido en un pilar fundamental para su construcción permitiendo el acceso a la información de forma flexible y confiable. 
así, el Gobierno puede dar inclusión, fiabilidad en la prestación de servicios y mejorar la calidad de vida de los ciudadanos.

\section{CONCLUSIÓN O CONSIDERACIONES FINALES}

El uso de las tecnologías de información a través de la interoperabilidad se ha hecho necesarias para diversos fines y propósitos, está constantemente presente en la vida cotidiana de los ciudadanos, ya sea como medio para informarse o realizar algún trámite, sin importar el estrato social que se tenga; sin embargo, todavía existen algunas limitantes en el uso en ciertas regiones por no contar con el internet; en ese sentido, a medida que la gente se involucra en el uso de las Tics se hace necesario analizar el potencial transformador e innovador que se les atribuye a estas herramientas. Asimismo, existe poco cumplimiento en los ámbitos de interacción, transacción, integración y participación, debido a que los portales de interoperabilidad de la gestión documental provinciales y municipales cumplan con las interacciones institucionales, ocasionando al usuario retraso en realizar sus trámites, contribuciones, aportaciones y obligaciones desde el portal web puesto que el usuario del siglo XXI, quiere todas las facilidades posibles a través de la tecnología.

La interoperabilidad es ineludible si se quiere lograr éxito en las estrategias de Gobierno electrónico, la organización de las ideas, las definiciones y las recomendaciones de la interoperabilidad requieren una estructuración nacional adecuada que permita su adopción ordenada y sin ambigüedades, el Estado debe implementar la plataforma de interoperabilidad a fin de que todas las entidades puedan cumplir.

Según Alarcón, (2020) la tendencia digital significa menos trabajo para los profesionales en la gestión documental; muchas organizaciones se entusiasman con la implementación de medidas que les permitirán gestionar su documentación digital, además la actualización de la normativa que facilita a las entidades generar documentos digitales también está a la orden del día, pero hay que tener mucho cuidado para no dejar de lado la gestión documental, los documentos físicos, también deben pasar a un repositorio con acceso limitado y, por último, un disco donde se conservará la documentación con carácter permanente; es por este motivo que hablamos de preservación digital.

La Unesco la define como «los procesos destinados a garantizar la accesibilidad permanente de los objetos digitales». Cuando trabajamos con documentos físicos pensamos en la manera de evitar el deterioro del papel, lo mismo ocurre con los 
documentos digitales: aunque no hablamos de papel, sí hablamos, por ejemplo, de versiones de softwares para acceder al documento (la versión del pdf, WordPerfect o Microsoft Word, entre otros), no debemos descuidar el volumen documental digital que estamos generando. Nos hemos acostumbrado a pensar en la eliminación documental porque sentimos el costo que implica tenerlo en un ambiente ordenado, sin embargo, si desde un inicio no aplicamos la gestión documental en la documentación digital que las entidades están generado, el costo de servidores y de personas buscando en los servidores será mucho mayor.

El Modelo de Gestión Documental, debió funcionar en todas las instituciones desde finales del 2018, sin embargo con la presencia del Covid-19 ha permitido acelerar algunos aspectos como la implementación de la mesa de partes virtual y el uso de la firma digital, es cuestión de tiempo para que la interoperabilidad funcione al 100\% entre las entidades del Estado a través de la PIDE, en el cual debemos desarrollar políticas para el intercambio de información digital y utilizar la información, el Estado debe mirar hacia una plataforma conjunta de información virtual; esperemos que la transformación digital sea una realidad en el Perú.

\section{LISTA DE REFERENCIAS}

1. Alvarado, P., Cevallos, P., Moran, E., \& Preciado, L. (2019). Modelo de gobierno electrónico peruano: servicios para el ciudadano en los portales de los gobienos regionales. 1-16. https://doi.org/10.33262/visionariodigital.v3i3.656

2. Alvarenga, A., Matos, F., Godina, R., João., M, (2020) Digital Transformation and Knowledge Management in the Public Sector. 1649-026 Lisboa, Portugal; arcfa@iscte-iul.pt. http://doi:10.3390/su12145824

3. Alarcón, E., (2020) La implementación del modelo de gestión documental en las entidades del Estado. http://www.revistaotlet.com/articulo-mgd-peru/

4. Arango, X., Estrada, O., Hernández, J. (2018). Análisis de los Potales Web en la Gestión Gubernamental de los Municipios del Estado de Nuevo León, México. Estudios de Administración, 3-19. ISSN 0717-0653 versión impresa | ISSN 07170616 versión en línea 
5. Araujo, S., Vargas, M., (2020). La interoperabilidad en el marco del Gobierno Digital. Revista Electronica Conocimiento Libre y Licenciamiento ( CLIC), Merida Venezuela. ISSN: 2244-7423

6. Bonal, J., \& Ortego, M., (2020). Los procesos de gestión de documentos en la Ley General de Archivos de México: análisis de contenido desde una perspectiva cuantitativa. Investigación bibliotecológica,vol. 34, núm. 85, octubre/diciembre, 2020, México, ISSN: 2448-8321 pp. 179-197

7. Cerrillo, A., Casadeús, A., (2018) El impacto de la gestión documental en la transparencia de las Administraciones públicas: la transparencia por diseño. GAPP. Nueva Época - N.o 19, mayo 2018 - ISSN: 1989-8991 - DOI: 10.24965/gapp.v0i19.10515 - Págs. 6-16

8. Cusihuamán, G., Alarcón, J., Ontiveros, W. (2020). Tecnologías de la información y comunicación, interculturalidad y desarrollo rural en la provincia de la Unión, Arequipa- Perú. Creative Commos, 15-29.h http://www doi:10.30827/publicaciones. v50i2.13940

9. Crespo, F., (2019). La Descripción Archivística en el Marco de la Gestión Documental por Procesos. Revista Española de Documentación Científica, 42 (4): e248. https://doi.org/10.3989/redc.2019.4.1632

10. De Grande, P. (2020). Gobierno Electrónico y Accesibilidad: Disponibilidad de Servicio en Plataformas Online Estatles de la Argentina . Revista Digital FCEUNLP, 4-14. https://doi.org/10.24215/23143738e 051

11. Díaz, M,.(2018) . Building digital government strategies. Principles and practices (Construyendo estrategias de gobierno digital. Principios y prácticas) Reseña [Reseña de libro] Investigación y Ciencia de la Universidad Autónoma de Aguascalientes,26(73),97-98.

http://www.redalyc.org/articulo.oa?id=67454781012

12. Diaz, A., Gonzales, A., (2020) La gestión documental en función de la gobernanza De la información. El caso de la Universidad Central "Marta Abreu” de las Villas. SAPIENTIAE: Revista de Ciencias Sociais, Humanas e Engenharias, vol. 
6, núm. 1, 2020 Universidade Óscar Ribas, Angola. http://www.redalyc.org/articulo.oa?id=572763612007

13. Eito-Brun, R.; Calleja, C. (2020). La gestión documental en los modelos de gobernanza TIC: presencia y visibilidad de la normativa internacional en el modelo de referencia COBIT. Revista Española de Documentación Científica, 43 (3), e272. https://doi.org/10.3989/redc.2020.3.1666

14. Febles A., Gonzáles, A. (2020). Ideas Iniciales del esquema nacional de interoperabilidad . Revista Cubana de Transformación Digital para el Gobierno electrónico en Cuba, 23-37.

15. Herrera-Cubides, J., Gaona- García, P., Sanchez-Alonso, S., (2018) Linked Data: qué sucede con la heterogeneidad y la interoperabilidad. http://www.redalyc.org/articulo.oa?id=84958001013

16. López, S. (2019). Gestión Documental: buena practica para reducir el consumo de papel en apoyo al desarrollo sostenible. GECONTEC, 78-92. ISSN 2255-5648

17. Lei, M., (2019) Interoperabilidad. Organización del conocimiento 46 (2): 122-146. 70 referencias. DOI: 10.5771 / 0943-7444-2019-2-122

18. Moreira-Mera, M., Hidalgo-Ávila, A., (2020). Gobierno electrónico en el Ecuador. Pol. Con. (Edición núm. 48) , pp. 944-961. https://doi 10.23857/pc.v5i8.1635

19. Naser, A., \& Concha, G. (2011). El gobierno electronico en la gestión públicaCEPAL. Santiago de Chile: Naciones Unidas.

20. Nations, U. (2018). E-Government Survey. New York.

21. Organización para la Cooperación y el Desarrollo Económico (OCDE). (2016). Gobierno Digital. En Políticas de banda ancha para América Latina y el Caribe: Un manual para la economía digital (389-421). Publicaciones OCDE. París. Disponible en: https: //n9.cl/i77q

22. Obukhov, A., Krasnyanskiy, M., Nikolyukin, M., (2020) Algoritmo de adaptación del sistema de gestión de documentos electrónicos basado en tecnología de 
aprendizaje automático. Universidad Técnica Estatal de Tambov, 106 Sovetskaya Street, Tambov 392000, Rusia. https://doi.org/10.1007/s13748-020-00214-2

23. Przeybilovicz, E., Cunha, M., \& Souza, F. (2018). The use of information and communication technology to characterize munipalities: who they are and what they need to develop e-government and smart city initiatives. RAP, 630-649. http://dx.doi.org/10.1590/0034-7612170582

24. Perpinyà, R., Cid-Leal, P., (2018) Los portales de archivos españoles: transparencia, interoperabilidad y orientación a los usuarios. Revista Española de Documentación Científica, $41 \quad$ (3): e212. https://doi. org/10.3989/redc.2018.3.1507

25. Presidencia de Consejo de Ministros (2020). Plataforma Digital de Gestión Documental. https://www.gob.pe/744-presidencia-del-consejo-de-ministroscero-papel

26. Rodríguez-Cruz, Y., (2020). La dimensión informacional de la Administración Pública para la Gobernanza y el gobierno “electrónico”y “abierto” RNPS: 2246 - ISSN: 2411-9970

27. Rubio-Núñez, R., Valencia, L., Peña-Cheng, L., Rodríguez-Muñoz, E. (2018). Importancia de la Gestión Tecnológica en los Gobiernos Municipales Mexicanos. Gestión de las Personas y Tecnología, 1-10. Obtenido de http://www.redalyc.org/articulo.oa?id=477857553008

28. Sáenz, A. (2019). La preservación digital en Colombia: un analisis dede la perspectiva normativa. Interam Biblotec Medellin, 87-97.

29. Sant, A., Durant, A., Zamora, C., Alberca, H. (2020). Análisis de la Adhesion a la plataforma de interoperabiidad del Estado en la Municipalidad de Lima- Perú. ADMPG, 1-12. http:// doi: 10.5212/Admpg.v.10.15066.002

30. Toro-García, A., Gutiérrez- Vargas , C., Correa- Ortiz, L.(2019). Estrategia de gobierno digital para la construccion de Estados más transparentes y proactivos. 72-102. https://doi.org/10.22430/21457778.1235 
31. Trocel, A., (2020). El Procedimiento Administrativo Electrónico Como medio para alcanzar la Buena Administración en Venezuela. Revista de la Facultad de Derecho No 73. 2018-2019 ISSN: 0255-5328. Pp 56 - Pp. 77

32. Zambrano- Yépez, C., Vélez -Romero, X., Vélez -Romero, Y (2019). Ranking de Gobierno Electrónico en los GAD provinciales de Ecuador y municipales de Manabí. Cientifica Dominio de las Ciencias, 355-374. http:// doi: $10.23857 /$ dc.v5i3.940 